\title{
TRANSTORNOS ALIMENTARES: O PAPEL DOS ASPECTOS CULTURAIS NO MUNDO CONTEMPORÂNEO
}

Leticia Langlois Oliveira*

Claúdio Simon Hutz

\begin{abstract}
RESUMO. Os transtornos alimentares são patologias caracterizadas primordialmente pelo medo mórbido de engordar. Vários são os fatores envolvidos tanto na etiologia quanto na manutenção e na gravidade dessas doenças, principalmente fatores individuais, familiares e culturais. Em relação a estes últimos, salienta-se o papel dos aspectos históricos, estéticos, midiáticos, transculturais, socioeconômicos, raciais e de gênero. Pesquisas atuais realizadas em diversas culturas demonstram a existência de uma relação entre esses fatores e os índices de anorexia nervosa e bulimia nervosa. A busca por um padrão estético globalizado (magreza), segundo a literatura, tem um papel central no aumento do número de casos. O objetivo deste artigo é apresentar uma discussão teórica acerca dos aspectos culturais envolvidos no aumento do número de casos de transtornos alimentares. Este trabalho pretende também destacar a necessidade de que profissionais de diversas áreas estejam atentos para os perigos do padrão de beleza atual, a fim de potencializar intervenções preventivas.
\end{abstract}

Palavras-chave: Transtornos alimentares; aspectos culturais; padrões de beleza.

\section{EATING DISORDERS: THE ROLE OF CULTURAL ASPECTS IN THE CONTEMPORARY WORLD}

\begin{abstract}
Eating disorders are pathologies mainly characterized by the morbid fear of gaining weight. Several factors comprise the etiology, the endurance and severity of these illnesses such as patient's individuality, family and culture. About the latter, the historical, aesthetic, the media, transcultural, socioeconomic, racial and gender roles are underlined. Recent research performed in various cultural backgrounds has shown the existence of a relationship between these factors and the levels of anorexia nervosa and bulimia nervosa. The aim of this article is to show a discussion about the cultural aspects involved in the increase number of cases of eating disorders. This paper aims to underline the need of experts of various fields to be aware of the dangers of the present beauty standards, so that preventive interventions can be addressed.
\end{abstract}

Key words: Eating disorders; cultural aspects; beauty standards.

\section{TRANSTORNOS ALIMENTARIOS: EL PAPEL DE LOS ASPECTOS CULTURALES EN EL MUNDO CONTEMPORÁNEO}

\begin{abstract}
RESUMEN. Los transtornos alimentarios son patologías caracterizadas primordialmente por el miedo mórbido de engordar. Varios son los factores involucrados ya sea en la etiología cuanto en la manutención y en la gravedad de esas enfermidades: individuales, familiares y culturales. Con relación a esos últimos, se resalta el papel de los aspectos históricos, estéticos, midiáticos, transculturales, socio económicos, raciales y de género. Investigaciones actuales realizadas en diferentes culturas muestran la existencia de una relación entre esos factores y los índices de anorexia nervosa y bulimia nervosa. El objetivo de ese artículo es presentar una discusión a cerca de los aspectos culturales involucrados en el aumento del número de casos de transtornos alimentarios. Ese trabajo pretende también destacar la necesidad de que profesionistas de diversas áreas estean atentos para los peligros del patrón de belleza actual, con la finalidad de potencializar intervenciones preventivas.
\end{abstract}

Palabras-clave: Transtornos alimentarios; aspectos culturales; patrones de belleza.

Doutora em Psicologia. Autônomo do Consultório de psicologia, Brasil.

\# Professor titular no Programa de Pós-Graduação em Psicologia da Universidade Federal do Rio Grande do Sul, Bolsista I-A do CNPq. 


\section{TRANSTORNOS ALIMENTARES: CONSIDERAÇÕES GERAIS}

Os transtornos alimentares são quadros caracterizados por aspectos como medo mórbido de engordar, preocupação exagerada com o peso e a forma corporal, redução voluntária do consumo nutricional com progressiva perda de peso, ingestão maciça de alimentos seguida de vômitos e uso abusivo de laxantes e/ou diuréticos (Melin \& Araújo, 2002). São patologias graves e de prognóstico reservado, que provocam elevados índices de letalidade e levam a limitações físicas, emocionais e sociais (Abreu \& Cangelli Filho, 2005)

Sabe-se que os transtornos alimentares afetam predominantemente a população jovem. Estima-se a incidência entre $0,5 \%$ e $1 \%$ para anorexia nervosa e $1 \%$ e $3 \%$ para bulimia nervosa em adolescentes do sexo feminino, números que são questionáveis, pelo fato de que apenas os casos mais graves chegam ao conhecimento médico. Além disso, distúrbios não especificados e síndromes parciais, isto é, formas de transtornos alimentares que não preenchem os critérios necessários para o diagnóstico de anorexia nervosa ou bulimia nervosa são bem mais frequentes, ocorrendo com $5 \%$ a $10 \%$ de jovens do sexo feminino (Melin \& Araújo, 2002).

Em relação à etiologia da anorexia nervosa e bulimia nervosa, são crescentes as evidências da interação de diversos fatores tanto na gênese mas quanto na manutenção dos transtornos (Morgan, Vecchiatti \& Negrão, 2002). Atualmente, a maioria dos pesquisadores trabalha com um modelo multidimensional, que reconhece fatores de risco de diversos níveis, como culturais, individuais e familiares (Abreu \& Cangelli Filho, 2005). Neste artigo são enfatizados alguns aspectos culturais relacionados ao aumento do número de casos de transtornos alimentares nas últimas décadas.

\section{MÉTODO}

O presente estudo compreendeu três fases distintas: (a) revisão sistemática da literatura; (b) avaliação crítica de artigos empíricos e da bibliografia; (c) síntese dos resultados. Na revisão da literatura realizou-se exaustiva pesquisa bibliográfica on-line, utilizando-se como fontes de busca as bases do Google Acadêmico, MedLine, LILACS e Scielo. Os termos descritores foram: "anorexia nervosa," "bulimia nervosa", "eating disorders", “culture”, "social" $e$ "globalization". Assim, neste estudo, inicialmente definiu-se a área de interesse e localizaram-se as investigações, e os achados foram sintetizados, apresentados e discutidos de forma ampla e introdutória.

\section{ASPECTOS CULTURAIS DOS TRANSTORNOS ALIMENTARES: PADRÕES DE BELEZA.}

Constata-se, na leitura sobre o histórico da anorexia nervosa e da bulimia nervosa, que estas não são doenças novas. Apesar de serem consideradas síndromes das sociedades ocidentais modernas, há registros esporádicos de patologias similares aos transtornos alimentares datados de vários séculos atrás. Ademais, a prevalência de dessas doenças tem crescido de forma significativa. Pesquisas mostram que a incidência praticamente dobrou nas últimas duas décadas (Morgan, Vecchiatti \& Negrão, 2002). Os estudiosos da área passaram então a realizar investigações sobre a associação entre os padrões de beleza estipulados pela cultura ocidental e o incremento do número de casos. O ideal de magreza vigente nestas sociedades é entendido pela literatura como um dos fatores culturais centrais que contribuiriam para o aumento destes transtornos na pós-modernidade (Hercovici \& Bay, 1997).

O conceito de beleza sofreu inúmeras variações ao longo da história. Quanto ao padrão estético corporal, o belo é normatizado desde os primórdios dos regimes patriarcais. Todas as culturas se desenvolvem marcadas por modelos estéticos fortemente definidos (Hercovici \& Bay, 1997).

Hábitos e práticas alimentares são construídos com base em determinações socioculturais (Serra, 2001). Neste sentido, a economia é um dos aspectos influentes. Houve sempre uma contradição entre a oferta de alimentos e as formas corporais femininas que são valorizadas. Assim, em épocas nas quais os alimentos são escassos, a imagem feminina robusta é sinal de poder e opulência, enquanto em períodos nos quais os alimentos são oferecidos em abundância, como atualmente, a magreza representa autodisciplina e sucesso (Hercovici \& Bay, 1997).

Percebe-se, então, que as práticas alimentares e os padrões estéticos corporais caminham juntos. Mais do que tendência ou produto da disponibilidade alimentar, os modelos de beleza são indicativos de distinção social e sinalizadores das diferenças entre classes sociais (Carreteiro, 2005).

Neste sentido, especificamente em relação ao Brasil, desde a Independência até os dias atuais os modelos de beleza têm se modificado e se adaptado ao contexto econômico, social, político e histórico. No século XIX, por exemplo, quando a culinária primava 
pelo alto teor calórico, o padrão corporal feminino desejado era o de formas arredondadas e flácidas (Carreteiro, 2005).

A observação da evolução dos padrões estéticos demonstra, a partir dos anos 60 (com auge na atualidade), a construção de uma imagem feminina esquálida, materializada nas manequins e modelos, que vêm assumindo antropometrias cada vez menores. No começo dos anos 80, o incremento da busca pela magreza já podia ser percebido com clareza. Em uma pesquisa que comparava o peso e as formas corporais de candidatas do concurso "Miss América" e modelos da capa de uma famosa revista masculina do ano de 1959 ao ano de 1978, foi constatada uma mudança gradual no padrão estético. Os autores do estudo verificaram uma transição progressiva das formas corporais curvilíneas para um modelo corporal cada vez mais magro e sem formas definidas (Andrade \& Bosi, 2003).

O final do século XX e início do século XXI são marcados pelo culto ao corpo. Há uma busca pelo corpo perfeito realizada de maneira obsessiva, transformando-se em um estilo de vida para muitas pessoas, especialmente para mulheres das classes médias urbanas (Castilho, 2001). O preconceito contra a obesidade é muito intenso, e a magreza é ligada à imagem feminina de sucesso, perfeição competência, autocontrole e atratividade sexual (Andrade \& Bosi, 2003).

No mundo contemporâneo, a globalização e a mídia em todas as suas formas de expressão vêm desempenhando, como nunca antes, papéis estruturantes na construção deste modelo (Serra, 2001). Personalidades, atrizes e modelos esbeltas, com alta estatura e musculatura definida ditam o ideal corporal que "deve" ser seguido (Carreteiro, 2005).

Estudos demonstram dados preocupantes neste sentido. Por exemplo, o preconceito contra a gordura acomete as crianças desde a pré-escola e mulheres com sobrepeso têm mais dificuldade em conseguir emprego e bons salários do que aquelas que estão dentro do padrão estético valorizado (Puhl \& Brownell, 2001).

Assim, o ser humano é pressionado, de diversas formas, a concretizar, no próprio corpo, o ideal corporal da cultura na qual está inserido. A sociedade, principalmente a feminina, mais vulnerável aos ideais de beleza criados culturalmente, é pressionada para essa representação por castigos (críticas, desprezo, deboche) e gratificações (dinheiro, poder, admiração). Os ganhos secundários são muitas vezes tão poderosos que o indivíduo renuncia ao contato interno e perde a conexão com seu corpo real (Carreteiro, 2005).
O corpo se tornou um dos valores mais importantes no atual momento histórico. A indústria da estética corporal é hoje um dos maiores mercados da sociedade de consumo (Carreteiro, 2005). O avanço da tecnologia da beleza, através da mídia, modela subjetividades e impulsiona a indústria da magreza (Andrade \& Bosi, 2003). A publicidade aumenta o desejo de cada de ter um corpo semelhante ao que ela sugere de forma repetitiva, portanto, também o de poder transformá-lo. Em consequência, os indivíduos veem o corpo como instrumento que deve ser perfeito e passível de constante remodelagem (Carreteiro, 2005). O aumento no número de cirurgias plásticas estéticas é um bom exemplo da crescente insatisfação corporal e da busca de formas perfeitas. É notável também o crescente número de sites que incentivam as adolescentes a desenvolver ou manter transtornos alimentares, ensinando técnicas de purgação e "truques" supostamente milagrosos para atingir um estado severo de emagrecimento.

Segundo Cury (2005), esse padrão inatingível de magreza, amplamente difundido na mídia (televisão, revistas, cinema) e nos desfiles e comerciais, etc. já penetrou no inconsciente coletivo e aprisionou as pessoas dentro de si mesmas. Em consequência disso, mais de $98 \%$ das mulheres não se veem bonitas. As mulheres nunca foram tão expostas a ideais estéticos quanto hoje, era da tecnologia da produção de massa. Desta forma, mulheres passam para outras mulheres estes mandatos, escravizando-as e colocando-as em padrões rígidos (Serra, 2001).

Pode-se constatar então que atualmente há uma busca desmedida por esse padrão de beleza massificado que, na maioria dos casos, é biologicamente impossível de ser alcançado. Essa busca obsessiva acaba desfigurando, assim, a tênue linha divisória entre o cuidado saudável com o corpo e o sutil movimento de instalação de doenças (Andrade \& Bosi, 2003). Tal padrão estético é considerado pela literatura como central no aumento do número de casos de transtornos alimentares (Serra, 2001).

\section{ASPECTOS CULTURAIS DOS TRANSTORNOS ALIMENTARES: ACHADOS ATUAIS.}

Pesquisas realizadas em diversas culturas confirmam a existência de uma relação entre a exposição à mídia e as desordens alimentares. Estudos americanos e europeus indicam que $70 \%$ das mulheres se sentem com sobrepeso, embora sejam normais ou magras (Hercovici \& Bay, 1997). Como já descrito, tais índices podem, em parte, ser explicados pela exploração dos meios de comunicação dessa imagem 
de mulheres em estado de inanição como padrão estético, estando os ditames da moda absolutamente inadequados à realidade da grande maioria da população. Com a dificuldade de adquirir o peso baixo desejado por meio de dietas saudáveis e exercícios físicos, as pesquisas mostram que muitas adolescentes passam a desenvolver práticas inadequadas de controle de peso.

O padrão de beleza veiculado pelos meios de comunicação e pelo convívio social parece exercer um efeito marcante sobre as mulheres. Universitárias sem transtornos alimentares expostas ao modelo de corpo magro, a imagens neutras e a imagens de mulheres de peso normal/sobrepeso relataram que as mulheres magras eram tidas como mais atraentes e que a exposição ao modelo magro gerava um aumento de respostas afetivas negativas (culpa, depressão, infelicidade, vergonha) (Stice, Schupak-Neuberg, Shaw \& Stein, 1994).

Na mesma linha, em estudo realizado nas ilhas Fiji, Becker et al. (2002) avaliaram o impacto da exposição das adolescentes à televisão e as consequentes atitudes e comportamentos alimentares desses indivíduos. O estudo foi dividido em duas etapas: a primeira em 1995 e a segunda em 1998 - já com três anos de exposição à televisão. Os resultados mostraram que os indicadores de transtorno alimentar foram significantemente mais acentuados após 1998, demonstrando também maior interesse em perda de peso, o que sugere um impacto negativo da mídia.

Outra pesquisa que relatou uma forte relação entre exposição à mídia e desenvolvimento de transtornos alimentares foi realizada na Tanzânia. Entrevistas feitas com 214 mulheres de 13 a 30 anos constataram que aquelas com maior número e severidade de sintomas relacionados a esses distúrbios eram as que mais tinham acesso à televisão, à internet e ao cinema (Eddy, Hennessey \& Thompson-Brenner, 2007).

Em estudo realizado na Inglaterra (Bell, Lawton \& Dittmar, 2007), 87 garotas adolescentes foram divididas em três grupos: o primeiro foi exposto a modelos magras em vídeos de música, o segundo ouvia somente as músicas dos vídeos, sem a imagem, e ao terceiro foi dada uma lista de palavras neutras para serem memorizadas e evocadas. Foi constatado que no primeiro grupo houve um aumento significativo da insatisfação com o corpo, comparado às outras intervenções, demonstrando o impacto deste tipo de mídia na construção da imagem corporal de adolescentes.

A esse respeito, Blowers et al. (2003) concluíram que as mulheres jovens percebem como a maior pressão para serem magras a que estão sujeitas aquela exercida pelos meios de comunicação, os quais possuem forte associação com a pressão da família e do grupo de amigas. Destarte, a pressão difundida pelos meios de comunicação torna-se mais efetiva quando associada a outras fontes de pressão do cotidiano, como os pares e familiares.

Durante a adolescência, sabe-se que os amigos passam a ter influência importante na socialização. McCabe e Ricciardelli, (2006) sugerem que tanto a mídia quanto o grupo social (amizades) têm papel mais significante na insatisfação corporal entre os adolescentes do sexo masculino do que os pais. Adolescentes do sexo feminino encorajadas por amigas meninas têm mais chances de fazer dietas. Além disso, amigas do mesmo sexo exercem mais influência do que as mães em meninas na adolescência tardia, em relação a atitudes e comportamentos relacionados à alimentação. Mais ainda, o reforço social exercido pela família, pelo grupo de pares e pela mídia em adolescentes e adultas jovens para ter o corpo magro relaciona-se à presença de sintomas bulímicos e prediz o início de sintomas nesta população.

No Brasil, são poucas as pesquisas na área. O estudo de base populacional desenvolvido por Nunes, Olinto, Barros e Camey (2001) demonstrou que a percepção inadequada do peso corporal de jovens mulheres entre 12 e 29 anos de idade (sentir-se gorda) tem um papel mais importante na determinação dos comportamentos alimentares anormais do que o real índice de massa corporal (IMC).

Outro estudo, realizado em 1999, analisou revistas nacionais voltadas para o público adolescente. Foram coletadas algumas frases de reportagens sobre beleza e as autoras puderam constatar, também no país, a divulgação de um padrão estético que é imposto às adolescentes e compromete sua saúde física e mental (Santos \& Serra, 2003).

Assim, o medo de engordar intenso entre as adolescentes favorece o desenvolvimento de comportamentos de risco para os transtornos alimentares como a realização de dietas extremadas, atividades físicas em excesso, utilização de medicamentos diuréticos, laxantes e anorexígenos e provocação de vômitos (Andrade \& Bosi, 2003). Especialmente em relação às práticas inadequadas de controle de peso entre adolescentes, os dados são alarmantes. Estudos como o de Fiates e Salles (2001), que entrevistaram 221 mulheres universitárias no Estado de Santa Catarina, apontam que 22,17\% da amostra apresentavam alterações importantes no comportamento alimentar. 


\section{ASPECTOS CULTURAIS DOS TRANSTORNOS ALIMENTARES: FATORES TRANSCULTURAIS, SOCIOECONÔMICOS E RACIAIS}

Hoje sabe-se que os transtornos alimentares acometem diferentes classes sociais e variadas regiões culturais, porém há algumas décadas a literatura descrevia que os indivíduos acometidos por transtornos alimentares eram, quase em sua totalidade, meninas ocidentais, de raça branca e pertencentes a uma classe socioeconômica alta. Apesar de ser inquestionável que os índices de anorexia nervosa e bulimia nervosa vêm crescendo de forma alarmante entre estas adolescentes, hoje tais enfermidades parecem se estender sobre os limites de raça, sexo ou cor. Um exemplo desta afirmação é o Japão, onde antes da ocidentalização tais transtornos praticamente inexistiam e atualmente são muito frequentes (Hercovici \& Bay, 1997).

Neste sentido, alguns autores têm questionado a concepção estabelecida de que os transtornos alimentares atingem predominantemente mulheres caucasianas. Até o momento, poucos estudos se propõem a comparar diferentes culturas e grupos étnicos, e os resultados encontrados são contraditórios.

Comprovando os achados iniciais, Chen e Swalm (1998) encontraram maiores índices de insatisfação corporal em adolescentes americanas do que em adolescentes asiáticas. Na mesma linha, Nasser (1998) constatou que mulheres negras americanas, mesmo tendendo e ter um peso corporal mais elevado, preocupam-se menos com o fato de engordar do que as mulheres brancas do mesmo país.

Ainda nos Estados Unidos, um estudo realizado no estado de Minnesota mostrou que as adolescentes africanas e as miscigenadas relataram com mais frequência alta satisfação corporal (40,1 e 44,3\%, respectivamente), enquanto hispânicas e asiáticas declararam menos satisfação $(19,8$ e $20,0 \%$, respectivamente); e que as africanas expressaram quase três vezes mais satisfação corporal do que as caucasianas (Kelly et al., 2005).

Por outro lado, alguns pesquisadores da área concluem que mulheres pertencentes à raça negra apresentam maiores índices de temor à obesidade do que mulheres caucasianas, provavelmente pelo preconceito que sofrem por parte da população de raça branca (Smith, Thompson, Raczinsky \& Hilner, 1999). Não obstante, a maioria das pesquisas atuais aponta que a insatisfação com o corpo, a distorção da imagem corporal e a realização de dietas para perder peso e de exercícios físicos que visam ao emagrecimento ocorrem na mesma proporção para mulheres caucasianas, negras, latinas, índias e asiáticas. É crescente o número de relatos de transtornos alimentares em países em desenvolvimento e em diferentes etnias, o que comprova que o temor da obesidade já é universal no mundo globalizado (O’Dea, 1994).

Há também uma relação entre a mídia e a urbanização na predisposição aos transtornos alimentares. De fato, um maior grau de urbanização parece aumentar a chance dessas patologias. A urbanização levaria a uma maior exposição ao ideal de beleza - a magreza - por meio da mídia, além de propiciar mudanças de hábitos alimentares, sedentarismo e um maior número de pessoas com obesidade (Morgan, Vecchiatti \& Negrão, 2002).

Outro aspecto sociocultural que vem sendo discutido se refere à relação dos transtornos alimentares com o nível econômico. Na Austrália, um estudo mostrou que jovens de nível socioeconômico médio, comparados com aqueles de nível alto, tinham menos probabilidade de desejar um corpo mais magro do que o escolhido como o atual. Esse estudo sugeriu que jovens de alto nível socioeconômico são mais influenciados por fatores como a família ou a mídia (Wang, Byrne, Kenardy \& Hills, 2005). Em outra pesquisa realizada naquele país, porém, não foi encontrada diferença entre adolescentes do sexo feminino de baixo ou médio/alto nível socioeconômico nas medidas de imagem corporal e comportamentos alimentares (O’Dea, 1994). Já em avaliação seguinte, O'Dea e Caputi (2001) averiguaram que jovens de baixo nível socioeconômico, especialmente meninos, têm mais probabilidade de se verem como muito magros e de tentarem ganhar peso, comparados com os controles de alto nível socioeconômico.

Ainda em relação aos aspectos socioeconômicos, Fernandes (2007), em seu estudo de base populacional com estudantes de seis a 18 anos de escolas públicas e particulares de Belo Horizonte, constatou que a classe social é significativa na construção da imagem corporal. Os alunos brancos, aqueles cujos responsáveis tinham curso superior completo, os pertencentes às classes socioeconômicas mais altas e os de escolas particulares desejavam ser mais magros, enquanto os negros, os alunos cujos responsáveis tinham o $1^{\circ}$ grau completo $/ 2^{\circ}$ grau incompleto, os pertencentes às classes socioeconômicas mais baixas e os de escolas públicas desejavam ser mais gordos.

\section{ASPECTOS CULTURAIS DOS TRANSTORNOS ALIMENTARES: INFÂNCIA EM PERIGO.}

Essa percepção corporal disfuncional e a busca pelo corpo considerado perfeito não atingem apenas 
os adolescentes e adultos. As crianças aprendem desde cedo, com suas famílias e com o meio social, a valorizar o corpo magro, e muitas vezes, mesmo com peso ideal, relatam insatisfação com suas formas corporais, engajando-se em condutas para emagrecer (Castilho, 2001).

Sabe-se que mais ou menos aos dois anos, a criança já possui autopercepção e consegue reconhecer a sua imagem no espelho. O corpo, gradualmente, vai representando a sua identidade e, aos poucos ela passa a pensar sobre como os outros veem a sua aparência. Os pré-escolares vão aprendendo como a cultura na qual estão inseridos avalia diferentes características físicas. Sua imagem corporal vai se desenvolvendo na medida em que introjetam conceitos do que é valorizado como atraente. As crianças também formam imagens do que não é valorizado ou aceito, ou seja, de como não "deveriam" parecer (Castilho, 2001).

Pesquisas constatam que garotas de cinco anos de idade já expressam preocupações com seu corpo e possuem conhecimento sobre dietas alimentares (Abramovitz \& Birch, 2000). Na mesma linha, vários estudos realizados com crianças nos Estados Unidos e na Inglaterra têm revelado que a maioria das garotas entre sete e nove anos concorda que é ruim ser gorda. A metade expressa desejo de ser mais magra e cerca de um terço sente medo de se tornar gorda (Shapiro, Newcombe \& Loeb, 1997).

Também no Brasil os números preocupam. Um estudo desenvolvido na cidade de Porto Alegre mostrou que $38,1 \%$ de crianças com peso adequado se consideram "gordas", entre elas especialmente meninas de 11 anos de idade, com maior IMC (índice de massa corporal), menor autoestima e meninas que pensam que seus pais gostariam que fossem mais magras (Pinheiro \& Giugliani, 2006).

Também neste sentido, o estudo já citado de Fernandes (2007) apontou que a maioria dos estudantes apresenta insatisfação com o próprio corpo. Dos alunos entrevistados, $62,6 \%$ estavam insatisfeitos com o próprio corpo, embora mais de $80 \%$ do total estivesse dentro do peso normal. Do total, 33,7\% gostariam de ser mais magros, 28,9\% gostariam de ser mais gordos e apenas $37,4 \%$ sentiam-se bem com o corpo.

\section{ASPECTOS CULTURAIS DOS TRANSTORNOS ALIMENTARES: GÊEERO.}

Embora as pesquisas constatem que pelo menos 95\% dos casos de anorexia nervosa e bulimia nervosa ocorrem em mulheres, percebe-se, neste estudo de
Fernandes (2007), um alto índice de insatisfação na população masculina infantil. Os transtornos alimentares apresentam uma distribuição assimétrica quanto ao gênero, assim como outras poucas patologias psiquiátricas. Esta diferença entre os sexos pode estar relacionada especialmente aos fatores socioculturais já descritos, mas também a aspectos biológicos, bem como ao pouco interesse dado, no passado, ao estudo destas doenças na população masculina.

Uma das hipóteses mais aceitas para a maior incidência de anorexia nervosa e bulimia nervosa no sexo feminino é que os homens não sofrem tanta pressão social para a magreza e aparência física e costumam aceitar com mais facilidade a sua imagem corporal. Ademais, há o consenso de que a satisfação com a imagem corporal feminina é pior que a masculina e que os homens percebem seus corpos com menos distorção (Cury, 2005). Para eles, a satisfação é conquistada através de uma série de fontes, como amizades e esportes, e a beleza não ocupa um papel tão central; já para muitas mulheres, a autoestima, o bem-estar subjetivo e a felicidade parecem estar diretamente relacionados ao ideal de corpo. Assim, a magreza muitas vezes é buscada pelas meninas através de comportamentos de risco que levam à instalação dos transtornos alimentares (Melin \& Araújo, 2002).

Não obstante, na última década houve um aumento considerável dessas patologias em homens, o qual é relacionado na literatura às transformações culturais da atualidade. Para o sexo masculino há também padrões socioculturais impostos: os de um corpo forte e definido. A dieta e outros comportamentos de risco são adotados por eles como uma forma de se adaptar a esse modelo de beleza, onde a gordura não é permitida (Morgan, Vecchiatti \& Negrão, 2002). Constata-se, atualmente, que a insatisfação corporal é um problema que afeta ambos os sexos, embora de formas diferentes. Há uma subestimação da própria condição física nos homens e a superestimação nas mulheres; ou seja, as meninas desejam ser mais esbeltas, e os meninos, ter corpos mais musculosos (Pinheiro \& Giugliani, 2006).

Essa distorção está comumente associada à anorexia nervosa e bulimia nervosa nas mulheres e à vigorexia nos homens. A vigorexia ou transtorno dismórfico muscular é a distorção da imagem corporal de maneira inversa. Os vigoréxicos se percebem como fracos e com pouca massa muscular mesmo que estejam exageradamente fortes e musculosos. Assim como nos transtornos alimentares predominantemente femininos, esses indivíduos apresentam um medo intenso da gordura. Para conquistarem a forma 
desejada frequentemente fazem uso de suplementos alimentares, anabolizantes e dietas hiperproteicas e realizam atividades físicas em excesso (Melin \& Araújo, 2002).

\section{CONSIDERAÇÕES FINAIS}

Os transtornos alimentares são patologias graves e de etiologia complexa. Entre os especialistas prevalece um entendimento multifatorial (Abreu \& Cangelli Filho, 2005), em que os aspectos culturais têm significativa importância. Constata-se que o ideal de magreza vigente é um dos importantes fatores culturais que contribuem para o incremento destes transtornos na pós-modernidade. O campo de investigação sobre a maneira pela qual o mundo globalizado, a sociedade e a mídia veiculam padrões estéticos e, por consequência, dietas e hábitos alimentares inadequados, é bastante vasto (Serra, 2001), novo e pouco explorado pelos profissionais da área.

Considera-se fundamental a pesquisa sobre a influência da mídia no desenvolvimento dessas patologias, em vista do aumento da amplitude e gravidade de tais desordens. São muito preocupantes os dados sugeridos pelas pesquisas na área em relação ao alto grau de insatisfação corporal, aos índices de transtornos alimentares, à obesidade, a comportamentos alimentares de risco, ao uso de medicamentos anorexígenos e laxantes, entre outros, já em idade precoce.

Neste sentido, cabe refletir sobre a contradição apresentada pela sociedade atual especialmente às crianças e aos adolescentes: de um lado enfatiza a atividade física e a importância de manter hábitos alimentares saudáveis, através dos mais variados veículos de comunicação; e do outro, o capitalismo e a urbanização "ditam" a magreza extrema como ideal de beleza e, ao mesmo tempo, incentivam a ingestão de alimentos rápidos e calóricos (fast food), o que estimula o aumento dos índices de obesidade mundial (Hercovici \& Bay, 1997).

Acredita-se que mais pesquisas são necessárias para um maior entendimento da relação entre comportamentos alimentares inadequados/transtornos alimentares e aspectos culturais, mais especificamente socioeconômicos, transculturais e raciais, especialmente no Brasil. Salienta-se a importância da realização de estudos interdisciplinares, que criem e utilizem métodos adequados para a avaliação do impacto da cultura na imagem corporal e nas práticas alimentares, especialmente em crianças e adolescentes. Sugere-se o desenvolvimento de pesquisas de delineamento qualitativo, necessárias para elucidar com mais profundidade fenômenos complexos, como o aumento de casos de transtornos alimentares em homens, a maior incidência de bulimia nas cidades do que em áreas rurais e as representações culturais da magreza.

É relevante também enfatizar a ação preventiva que a mídia pode realizar diante de tal assunto. Embora os meios de comunicação, em sua grande maioria, estimulem o padrão esbelto de beleza, algumas empresas, redes de televisão e personalidades estão veiculando campanhas para alertar sobre os perigos dos transtornos alimentares e do exagero da valorização exacerbada da magreza.

Por fim, acredita-se que a família, os profissionais da saúde e educadores devem estar atentos aos seus filhos, pacientes e alunos, estabelecendo continuamente um clima de diálogo, cuidado e informação no que se refere aos comportamentos de risco para o desenvolvimento de transtornos alimentares. Além disso, devem estimular e valorizar, nas crianças e adolescentes, outros valores nobres que vão de encontro a esse ideal doentio de beleza.

\section{REFERÊNCIAS}

Abramovitz, B. A. \& Birch, L. L. (2000). Five-year-old girls' ideas about dieting are predicted by mothers' dieting. Journal of American Diet Association, 2(2), 310-307.

Abreu, C. N. \& Cangelli Filho, R. (2005). Anorexia nervosa nervosa e bulimia nervosa nervosa: a abordagem cognitivoconstrutivista de psicoterapia. Psicologia: Teoria e Prática, 7(1), 153-165.

Andrade, A. \& Bosi, M. M. (2003). Mídia e subjetividade: impacto no comportamento alimentar feminino. Revista de Nutrição, 16(1), 117-125.

Becker, A. E., Burwell, R. A., Gilman, S. E., Herzog, D. B. \& Hamburg, P. (2002). Eating behaviors and attitudes following prolonged exposure to television among ethnic Fijian adolescent girls. British Journal of Psychiatry, 180, 509-514.

Bell, B. T., Lawton, R. \& Dittmar, H. (2007). The impact of thin models in music videos on adolescent girls' body dissatisfaction. Body Image, 4, 137-145.

Blowers, L. C., Loxton, N. J., Grady-Flesser, M., Occhipinti, S. \& Dawe, S. (2003). The relationship between sociocultural pressure to be thin and body dissatisfaction in preadolescent girls. Eating Behaviors, 4(3), 229-244.

Carreteiro, T. A. (2005). Corpo e contemporaneidade. Psicologia em Revista, 11(17), 62-76.

Castilho, S. M. (2001). A imagem corporal. Santo André: ESETec Editores Associados.

Chen, W. \& Swalm, R. L. (1998). Chinese and American college students' body image: perceived body shape and body affect. Perceptual and Motor Skills, 87, 395-403. 
Cury, A. J. (2005). A ditadura da beleza e a revolução das mulheres. Rio de Janeiro : Sextante.

Eddy, K. T., Hennessey, M. \& Thompson-Brenner, H. (2007). Eating pathology in East African women - the role of media exposure and globalization. The Journal of Nervous and Mental Disease, 195(3), 196-202.

Fernandes, A. E. R. (2007). Avaliação da imagem corporal, hábitos de vida e alimentares em crianças e adolescentes de escolas públicas e particulares de Belo Horizonte. Dissertação de Mestrado não publicada. Programa de Pós-Graduação em Ciências da Saúde da Faculdade de Medicina. Universidade Federal de Minas Gerais. Belo Horizonte, MG.

Fiates, G. M. R. \& Salles, R. K. (2001). Fatores de risco para o desenvolvimento de distúrbios alimentares: Um estudo em universitárias. Revista de Nutrição, 14, 1-8.

Hercovici, C. \& Bay, L. (1997). Anorexia nervosa e bulimia nervosa: Ameaças à autonomia. Porto Alegre: Artes Médicas.

Kelly, A. M., Wall, M., Eisenberg, M. E., Story, M. \& NeumarkSztainer, D. (2005). Adolescent girls with high body satisfaction: Who are they and what can they teach us? Journal of Adolescent Health, 37, 391-396.

McCabe, M. P. \& Ricciardelli, L. (2006) "Who thinks I need a perfect body?" Perceptions and internal dialogue among adolescents about their bodies Sex Roles: A Journal of Research 9, 1-20.

Melin, P. \& Araújo, A. M. (2002). Transtornos alimentares em homens: Um desafio diagnóstico. Revista Brasileira de Psiquiatria, 24(3), 73-76.

Morgan, C. M., Vecchiatti, I. R. \& Negrão, A. B. (2002). Etiologia dos transtornos alimentares: Aspectos biológicos, psicológicos e socioculturais. Revista Brasileira de Psiquiatria, 24(3), 18-23.

Nasser, M. (1998). Culture and weight consciousness. Journal of Psychosomatic Research, 32, 573-577.

Nunes, M. A. A., Olinto, M. T., Barros, F. C. \& Camey, S. (2001). Influência da percepção do peso e do índice de massa corporal nos comportamentos alimentares anormais. Revista Brasileira de Psiquiatria, 23(1), 21-27.

O'Dea, J. (1994). Food habits, body image and self-esteem of adolescent girl from disadvantaged and non-disadvantaged backgrounds. Australian Journal of Nutrition and Dietetics, 51, 74-78.
O'Dea, J. A. \& Caputi, P. (2001). Association between socioeconomic status, weight, age and gender, and the body image and weight control practices of 6- to 19-year-old children and adolescents. Health Education Research, 16, 521-532.

Pinheiro, A. P. \& Giugliani, E. R. J. (2006). Quem são as crianças que se sentem gordas apesar de terem peso adequado? Jornal de Pediatria, 82(3), 13-17.

Puhl, R. \& Brownell, K. (2001). Bias, discrimination, and obesity. Obesity Research, 9(12), 788-805.

Santos, E. M. \& Serra, G. M. A. (2003). Saúde e mídia na construção da obesidade e do corpo perfeito. Ciência \& saúde coletiva, 8(3), 691-701.

Serra, G. M. A. (2001). Saúde e nutrição na adolescência: $O$ discurso sobre dietas na Revista Capricho. Dissertação de Mestrado não publicada. Escola Nacional de Saúde Pública. Fundação Oswaldo Cruz. Rio de Janeiro, RJ.

Shapiro, S., Newcombe, M. \& Loeb, T. B. (1997). Fear of fat, disregulated-restrained eating, and body-esteem: Prevalence and gender differences among eight to ten years old children. Journal of Clinical Child Psychology, 26, 358-365.

Smith, D. E., Thompson, J. K., Raczemsky, J. M. \& Hilner, J. E. (1999). Body image among men and women in a biracial cohort: The CARDIA study. International Journal of Eating Disorders, 25, 71-82.

Stice, E., Schupak-Neuberg, E., Shaw, H. E. \& Stein, R. I. (1994). Relation of media exposure to eating disorder symptomatology: An examination of mediating mechanisms. Journal of Abnormal Psychology, 103(4), 836-840.

Wang, Z., Byrne, N. M., Kenardy, J. A. \& Hills, A. P. (2005). Influences of ethnicity and socioeconomic status on the body dissatisfaction and eating behaviour of Australian children and adolescents. Eating Behaviors, 6, 23-33.

Recebido em 09/02/2009 Aceito em 16/05/2010
Endereço para correspondência:
Leticia Langlois Oliveira. Rua Vicente de Paula Dutra, 195, ap. 403, Bairro Praia de Belas, CEP 90110-200, Porto Alegre-RS, Brasil. E-mail: leticiaufrgs@ yahoo.com.br. 